\title{
Association of seasonal variation in the prevalence of metabolic syndrome with insulin resistance
}

\author{
Fumihiko Kamezaki ${ }^{1,2}$, Shinjo Sonoda ${ }^{1}$, Sei Nakata ${ }^{1}$, Yoshitaka Muraoka ${ }^{1}$, Masahiro Okazaki ${ }^{1}$, \\ Masahito Tamura ${ }^{1}$, Haruhiko Abe ${ }^{1}$, Masaaki Tekeuchi ${ }^{1}$ and Yutaka Otsuji ${ }^{1}$
}

The aim of this study was to examine the hypothesis that seasonal variation in the prevalence of metabolic syndrome (MetS) is associated with increased insulin resistance. Among 840 Japanese male workers who were evaluated using the homeostasis model assessment of insulin resistance (HOMA-IR) in June (summer) 2010, we prospectively studied a total of 758 subjects (40-65 years of age) who underwent an assessment in December (winter) 2010. MetS was defined according to the criteria proposed by the International Diabetes Federation (IDF) and the Japanese Society of Internal Medicine (JSIM). The median level of HOMA-IR in the study subjects was 0.84 (interquartile range: $0.60-1.19$ ). The prevalence rates of IDF- and JSIM-MetS significantly increased from 12.4 and $9.6 \%$ in the summer to 16.6 and $13.3 \%$ in the winter, respectively (each $P<0.05$ ). Our data suggest that these increases are mainly due to increases in blood pressure (BP) and glucose during the winter assessment. The prevalence rates of IDF-MetS in the first, second, third and fourth quartiles of HOMA-IR were $1.1,5.8,14.3$ and $29.1 \%$ in the summer and $3.1,10.6,21.9$, and $31.3 \%$ in the winter, respectively. Similar results were obtained when using the JSIM criteria. In the third quartile, the frequency of elevated BP increased from $42.4 \%$ in the summer to $61.2 \%$ in the winter $(P<0.05)$, and these values were mainly correlated with significant variations in IDF- and JSIM-MetS prevalence rates. This study demonstrates that seasonal variation in MetS prevalence is associated with mildly to moderately increased insulin resistance in middle-aged Japanese men.

Hypertension Research (2013) 36, 398-402; doi:10.1038/hr.2012.197; published online 13 December 2012

Keywords: blood pressure; homeostasis model assessment of insulin resistance; metabolic syndrome; seasonal variation

\section{INTRODUCTION}

Metabolic syndrome (MetS) is a possible cause of type 2 diabetes and atherosclerotic cardiovascular disease worldwide. ${ }^{1,2}$ MetS consists of atherogenic dyslipidemia, elevation in blood pressure (BP), and increased glucose concentration as well as a general prothrombotic and proinflammatory condition. ${ }^{3}$ MetS can be clinically diagnosed using several simple measurements including waist circumference (WC), triglyceride (TG) levels, high-density lipoprotein (HDL) cholesterol levels, BP and fasting glucose levels. ${ }^{4,5}$ Evidence suggests that these metabolic parameters can show seasonal variations, and these variations usually tend to be on the higher end during the fall and winter than in the spring and summer. ${ }^{6-10}$ Seasonal variations in these metabolic parameters could be partially driven by gene variation, lifestyle factors such as diet, physical activity and sleep duration, climate conditions such as air temperature and light exposure, and mood changes such as depressive symptoms. ${ }^{11-18}$ These findings indicate that seasonal variations in metabolic parameters may affect the assessment of MetS. A recent study demonstrated the seasonal variation of MetS prevalence in Japanese men, but the underlying mechanisms involved with seasonal variation and the associated clinical implications remain unknown. ${ }^{19}$ Ample evidence suggests that increased insulin resistance is a pathophysiological basis for MetS. However, little is known about the relationship between seasonal variations in metabolic parameters and increased insulin resistance. Therefore, we hypothesized that seasonal variation in MetS prevalence is associated with increased insulin resistance. To test this hypothesis, we assessed Japanese workers using periodic health assessments.

\section{METHODS}

\section{Study subjects}

Among 969 male workers (40-65 years of age) who underwent a health checkup in June (summer) 2010, 840 subjects (86.7\%) provided written informed consent to participate in this study and thus were evaluated using the homeostasis model assessment of insulin resistance (HOMA-IR). After excluding subjects who did not undergo a health checkup in December (winter) $2010 \quad(n=76)$ and subjects who self-reported the use of antidiabetic drugs $(n=6)$, which could interfere with accurate evaluations

${ }^{1}$ Second Department of Internal Medicine, School of Medicine, University of Occupational and Environmental Health, Kitakyushu, Japan and ${ }^{2}$ Department of Emergency Medicine, School of Medicine, University of Occupational and Environmental Health, Kitakyushu, Japan

Correspondence: Dr F Kamezaki, Department of Emergency Medicine, School of Medicine, University of Occupational and Environmental Health, 1-1 Iseigaoka, Yahatanishi-ku, Kitakyushu 807-8555, Japan

E-mail: kamezaki@med.uoeh-u.ac.jp

Received 22 July 2012; revised 24 September 2012; accepted 26 September 2012; published online 13 December 2012 
using HOMA-IR, a total of 758 subjects (researchers, technical experts or clerks) were included in the study. At each checkup, demographic data and health information were collected using self-administered questionnaires. Smoking habits and alcohol intake were dichotomized as current smoker and/or drinker or not current smoker and/or drinker. All participants were subjected to a physical examination assessing height, weight, WC and BP. WC was taken as the circumference at the umbilicus using a flexible anthropometric tape, and resting BP was measured from a sitting position by trained technicians using a standard mercury sphygmomanometer. Venous blood samples were collected from each subject after fasting for $9 \mathrm{~h}$ or overnight. Using automated measurement devices, serum levels of TG (enzymatic method; Determiner C-TG, Kyowa Medex, Tokyo, Japan) and HDL cholesterol (direct method; Determiner L HDL-C, Kyowa Medex), and plasma glucose levels (hexokinase method; Quick auto II GLU-HK, Shino-test, Kanagawa, Japan) were measured.

This study was conducted in accordance with the principles expressed in the Declaration of Helsinki and was ethically approved by the institutional committee of the Nuclear Science Research Institute of the Tokai Research and Development Center at the Japan Atomic Energy Agency.

\section{Definition of MetS}

In this study, MetS was defined according to the criteria proposed by six major world organizations including the International Diabetes Federation (IDF) and the joint committee of eight Japanese medical societies including the Japanese Society of Internal Medicine (JSIM)., 4, 20,21 The IDF criteria are as follows: subjects with three or more of the following components are considered to have IDF-MetS: (1) elevated WC defined as WC $\geqslant 85 \mathrm{~cm}$ according to the JSIM criteria; (2) elevated TG defined as TG $\geqslant 150 \mathrm{mg}$ per $100 \mathrm{ml}$ or selfreported consumption of lipid-lowering medications; (3) reduced HDL cholesterol defined as HDL cholesterol $<40 \mathrm{mg}$ per $100 \mathrm{ml}$; (4) elevated BP defined as BP $\geqslant 130 / 85 \mathrm{mmHg}$ or self-reported consumption of antihypertensive drugs; (5) elevated glucose defined as having a fasting glucose level of $\geqslant 100 \mathrm{mg}$ per $100 \mathrm{ml}$. The sole criterion for JSIM diagnosis is abdominal obesity defined as a WC of $\geqslant 85 \mathrm{~cm}$. In addition, subjects with two or more of the following criteria are considered to have JSIM-MetS: (1) dyslipidemia defined as TG $\geqslant 150 \mathrm{mg}$ per $100 \mathrm{ml}$, HDL cholesterol $<40 \mathrm{mg}$ per $100 \mathrm{ml}$ or self-reported consumption of lipid-lowering medications; (2) elevated BP defined as BP $\geqslant 130 / 85 \mathrm{~mm} \mathrm{Hg}$ or self-reported consumption of antihypertensive drugs; (3) elevated glucose defined as having a fasting glucose level of $\geqslant 110 \mathrm{mg}$ per $100 \mathrm{ml}$. In this study, subjects who met the criteria in the summer, winter and both seasons were defined as having summer-, winterand summer-winter-MetS, respectively.

\section{Evaluation of HOMA-IR}

Fasting blood samples were transported to the same laboratory for analysis. Using an automated measurement device, the fasting insulin levels in the plasma of all subjects were measured by chemiluminescent immunoassay (ARCHITECT Insulin, Abbott Japan, Tokyo, Japan) in the summer 2010 checkup. In this assay, the interassay coefficients of variation were $<7 \%$, and the lower limit of detection was $1.0 \mu \mathrm{IU} \mathrm{ml}^{-1}$. HOMA-IR was calculated using the following equation: (fasting insulin $\left(\mu \mathrm{IU} \mathrm{ml}^{-1}\right) \times$ fasting glucose $(\mathrm{mg}$ per $100 \mathrm{ml}$ ))/405. HOMA-IR has been well correlated with estimates derived from the euglycemic clamp test. ${ }^{22,23}$

\section{Statistical analysis}

Continuous data are expressed as the median with the associated interquartile range (IQR) because the values do not display a normal distribution. All of the continuous data were log transformed to approximately normalize the distribution for the subsequent analyses. Categorical data are presented as absolute values and percentages. Comparisons of continuous variables were performed by using analysis of covariance with age as a covariate. Comparisons of categorical variables were evaluated using chi-squared tests. Analysis of variance (ANOVA) was used for multiple comparisons, and ANOVA tests were followed by the Tukey-Kramer post hoc test. $P$-values $<0.05$ were considered to be statistically significant. Statistical analysis was performed using the JMP
Statistical Discovery Software for Windows version 8 (SAS Institute, Cary, NC, USA).

\section{RESULTS}

Characteristics of study subjects

The characteristics of the study subjects ( $n=758$, median age 49 years (IQR: 44-55)) during the summer assessment are shown in Table 1. Some study participants were receiving medications for hyperlipidemia $(n=55,7.3 \%)$ and hypertension $(n=87,11.5 \%)$. Current smokers and drinkers comprised 23.2\% $(n=176)$ and $46.0 \%$ $(n=349)$ of the study population, respectively. Of the clinical and demographic parameters shown in Table 1, systolic and diastolic BP showed significant seasonal variation. Systolic and diastolic BP levels increased from $126 \mathrm{~mm} \mathrm{Hg}$ (IQR: $120-132 \mathrm{~mm} \mathrm{Hg}$ ) and $74 \mathrm{~mm} \mathrm{Hg}$ (IQR: $70-80 \mathrm{~mm} \mathrm{Hg}$ ) in the summer to $130 \mathrm{~mm} \mathrm{Hg}$ (IQR: $122-$ $136 \mathrm{~mm} \mathrm{Hg}$ ) and $76 \mathrm{~mm} \mathrm{Hg}$ (IQR: $72-82 \mathrm{~mm} \mathrm{Hg}$ ) in the winter, respectively (each $P<0.05$ ).

\section{Seasonal variations in MetS prevalence and components}

All subjects were assessed for variables of MetS components during the summer and winter checkups. The seasonal variations in MetS prevalence and the associated metabolic parameters are listed in Table 2. The prevalence rates of IDF- and JSIM-MetS in this study

\section{Table 1 Characteristics of study subjects}

\begin{tabular}{|c|c|}
\hline Characteristic & $n=758$ \\
\hline Body mass index, $\mathrm{kg} \mathrm{m}^{-2}$ & $23.5(21.7-25.3)$ \\
\hline Total cholesterol, mg per $100 \mathrm{ml}$ & 215 (194-236) \\
\hline LDL cholesterol, ${ }^{a}$ mg per $100 \mathrm{ml}$ & $130(112-150)$ \\
\hline Waist circumference, $\mathrm{cm}$ & $83.1(78.4-88.0)$ \\
\hline Triglyceride, mg per $100 \mathrm{ml}$ & $100(73-145)$ \\
\hline HDL cholesterol, mg per $100 \mathrm{ml}$ & $63(54-75)$ \\
\hline Systolic blood pressure, $\mathrm{mm} \mathrm{Hg}$ & $126(120-132)$ \\
\hline Diastolic blood pressure, $\mathrm{mm} \mathrm{Hg}$ & $74(70-80)$ \\
\hline Fasting glucose, mg per $100 \mathrm{ml}$ & $89(84-94)$ \\
\hline Lipid-lowering medication, $n(\%)$ & $55(7.3)$ \\
\hline Antihypertensive medication, $n(\%)$ & $87(11.5)$ \\
\hline Current smoking, $n(\%)$ & $76(23.2)$ \\
\hline Current drinking, $n(\%)$ & $349(46.0)$ \\
\hline
\end{tabular}

Abbreviations: HDL, high-density lipoprotein; LDL, low-density lipoprotein.

Data are expressed as median with interquartile range (IQR), or number (\%).

LDL cholesterol levels were evaluated by a direct measurement.

Table 2 Seasonal variations in MetS prevalence and components

\begin{tabular}{lrrr}
\hline & Summer & Winter & P-value \\
\hline IDF-MetS prevalence, \% & 12.4 & 16.6 & $<0.05$ \\
JSIM-MetS prevalence, \% & 9.6 & 13.3 & $<0.05$ \\
Elevated waist circumference, \% & 39.3 & 41.3 & 0.43 \\
Elevated triglyceride, \% & 27.8 & 28.6 & 0.73 \\
Reduced HDL cholesterol, \% & 3.0 & 2.0 & 0.19 \\
Elevated blood pressure, \% & 42.6 & 54.4 & $<0.05$ \\
Elevated glucose, \% & & & \\
$\quad$ Fasting glucose $\geqslant 100 \mathrm{mg}$ per $100 \mathrm{ml}$ & 11.0 & 14.6 & $<0.05$ \\
Fasting glucose $\geqslant 110 \mathrm{mg}$ per $100 \mathrm{ml}$ & 4.4 & 5.8 & 0.20 \\
Dyslipidemia, \% & 28.4 & 29.4 & 0.65
\end{tabular}

Abbreviations: HDL, high-density lipoprotein; IDF, International Diabetes Federation; JSIM, Japanese Society of Internal Medicine; MetS, metabolic syndrome. All data are expressed as percentage. 
significantly increased from $12.4 \%(n=94)$ and $9.6 \%(n=73)$ in the summer to $16.6 \%(n=126)$ and $13.3 \%(n=101)$ in the winter, respectively (each $P<0.05$ ). Although the frequencies of elevated WC, elevated TG, reduced HDL cholesterol, elevated glucose defined as fasting glucose $\geqslant 110 \mathrm{mg}$ per $100 \mathrm{ml}$ and dyslipidemia were comparable between the two seasons $(P=0.43, P=0.73, P=0.19, P=0.20$, and $P=0.65$, respectively), the frequencies of elevated BP and elevated glucose defined as fasting glucose $\geqslant 100 \mathrm{mg}$ per $100 \mathrm{ml}$ significantly increased from $42.6 \%(n=323)$ and $11.0 \%(n=83)$ in the summer to $54.4 \%(n=412)$ and $14.6 \%(n=111)$ in the winter, respectively (each $P<0.05$ ). Notably, the frequency of elevated BP had a large impact on the seasonal variation of MetS prevalence in our study subjects.

HOMA-IR levels in subjects with summer-, winter- and summerwinter-MetS

The median levels of fasting insulin and HOMA-IR in the study subjects were $3.7 \mu \mathrm{IU} \mathrm{ml}^{-1}$ (IQR: $2.7-5.2 \mu \mathrm{IU} \mathrm{ml}^{-1}$ ) and 0.84 (IQR: 0.60-1.19), respectively. Based on the IDF criteria, the median levels of HOMA-IR in subjects with summer- $(n=27,1.17$ (IQR: 0.92$1.66)$ ), winter- $(n=59,1.01$ (IQR: $0.80-1.32))$, and summer-winterMetS ( $n=67,1.31$ (IQR: 0.95-1.73)) were significantly higher than the median levels of HOMA-IR in subjects without MetS $(n=605$, 0.76 (IQR: 0.54-1.07)) (each $P<0.05)$ (Figure 1a). Furthermore, the levels of HOMA-IR were comparable in subjects with summer- and summer-winter-MetS $(P=0.98)$, whereas the levels of HOMA-IR were significantly lower in subjects with winter-MetS when compared with subjects with summer-winter-MetS $(P<0.05)$. When using the JSIM criteria, the median levels of HOMA-IR were comparable in subjects with summer-, winter- and summer-winter-MetS, and these levels were significantly higher than the HOMA-IR levels of subjects without MetS (Figure 1b).

\section{Association of HOMA-IR with seasonal variation in MetS prevalence}

To examine our initial hypothesis, we compared the influence of HOMA-IR levels on seasonal variations of MetS prevalence among four subgroups. Although the prevalence rates of IDF- and JSIMMetS in both seasons increased in patients in higher quartiles of HOMA-IR level and the prevalence data were higher in all quartiles during the winter when compared with the respective quartiles during the summer, the differences were not statistically significant except for the third quartile (Figures $2 \mathrm{a}$ and $2 \mathrm{~b}$ ): the rates of IDF- and JSIMMetS in the third quartile increased from 14.3 and $11.2 \%$ in the summer to 21.9 and $17.9 \%$ in the winter, respectively (each $P<0.05$ ). In the third quartile, seasonal frequencies of elevated WC, elevated TG, reduced HDL cholesterol, elevated glucose or dyslipidemia exhibited little change (data not shown), whereas the frequency of elevated BP increased from $42.4 \%(n=83)$ in the summer to $61.2 \%$ $(n=120)$ in the winter $(P<0.05)$, which was related to the significant variation in both the IDF- and JSIM-MetS prevalence (Figure 3 ). Otherwise, there were no significant seasonal variations in glucose levels in any of the quartiles based on the HOMA-IR level (data not shown).

\section{DISCUSSION}

The ultimate aim of MetS screening is to identify subjects who are at a high risk of developing type 2 diabetes and cardiovascular disease. ${ }^{24}$ Although MetS consists of various pathological conditions, MetS can be diagnosed clinically using several simple measurements including WC, TG levels, HDL cholesterol levels, BP and fasting glucose levels.
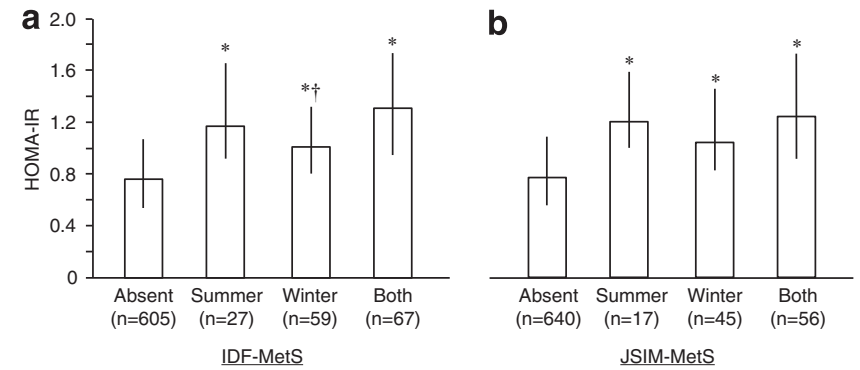

Figure 1 Homeostasis model assessment of insulin resistance (HOMA-IR) levels according to seasonal IDF-MetS (a) and JSIM-MetS (b). All data are expressed as the median with the associated interquartile range. ${ }^{*} P<0.05$ when compared with subjects without MetS, ${ }^{\dagger} P<0.05$ when compared with subjects with summer-winter-MetS by analysis of variance followed by the Tukey-Kramer post hoc test. IDF, International Diabetes Federation; JSIM, Japanese Society of Internal Medicine; MetS, metabolic syndrome.
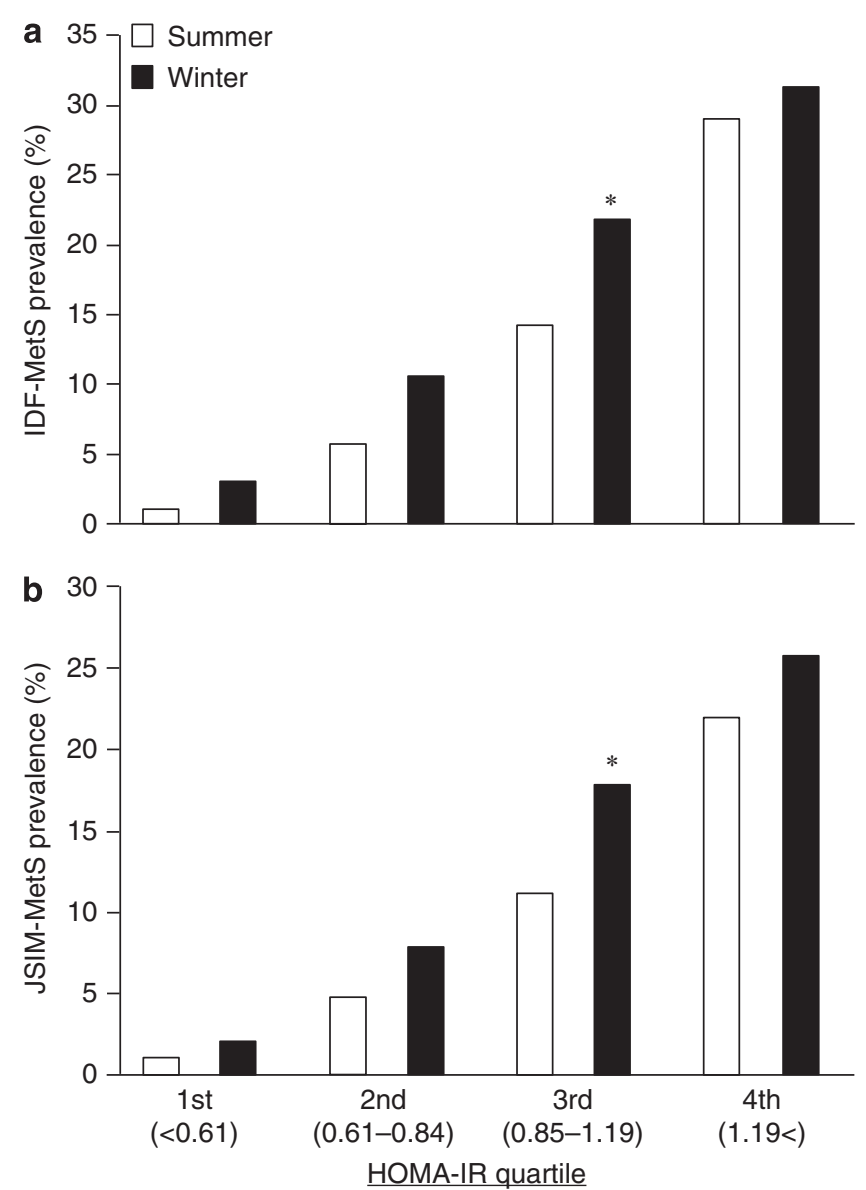

Figure 2 Seasonal prevalence rates of IDF-MetS (a) and JSIM-MetS (b) according to homeostasis model assessment of insulin resistance (HOMA-IR) levels. ${ }^{*} P<0.05$ when compared with summer-MetS as determined by the chi-squared test. IDF, International Diabetes Federation; JSIM, Japanese Society of Internal Medicine; MetS, metabolic syndrome. 


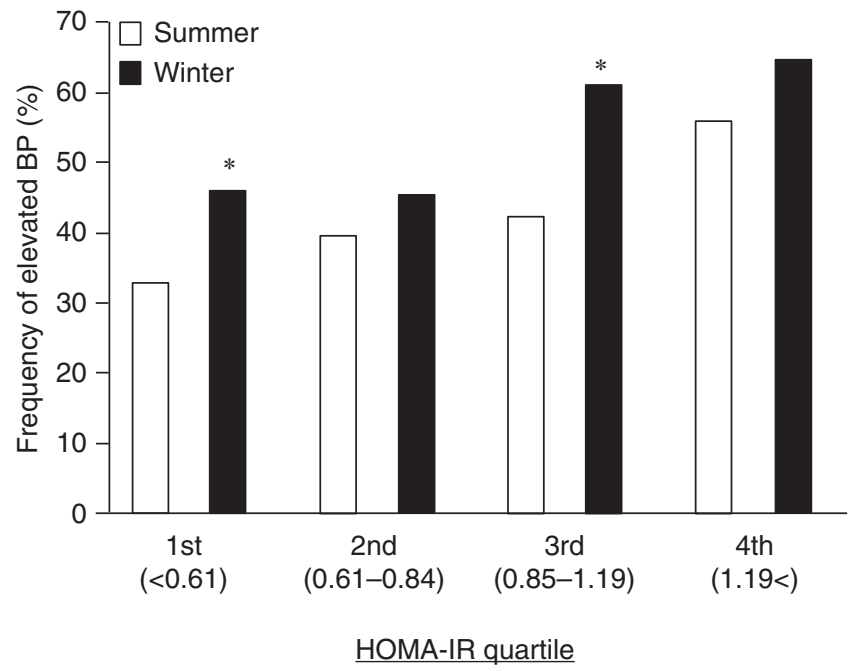

Figure 3 Seasonal frequency of elevated blood pressure (BP) according to homeostasis model assessment of insulin resistance (HOMA-IR) levels. ${ }^{*} P<0.05$ when compared with summer-MetS as determined by the chisquared test. MetS, metabolic syndrome.

However, despite accumulating evidence of seasonal variations in these metabolic parameters, seasonal variations are not included in the criteria used to diagnose MetS. These seasonal variations are usually higher in the fall and winter than in the spring and summer, and the incidence of cardiovascular disease is proportionately higher in the winter when compared with the other seasons. ${ }^{25,26} \mathrm{~A}$ recent report has suggested that seasonal variations in metabolic parameters may serve as a partial explanation for the higher incidence of cardiovascular disease during the winter. ${ }^{27}$ These findings indicate that seasonal variations in various metabolic parameters may be risk factors for cardiovascular disease. We recently demonstrated the seasonal variation of MetS prevalence in Japanese male workers. ${ }^{19}$ However, our study could not determine the underlying mechanism and clinical implication of this variation. Increased insulin resistance is one of the pathophysiological bases for MetS, and the HOMA-IR model is widely used to estimate insulin resistance in clinical practice. $^{28}$ In the present study, we examined the association between seasonal variation in MetS prevalence and insulin resistance evaluated by HOMA-IR.

There are several important findings in the present study. First, seasonal variation in MetS prevalence exists in middle-aged Japanese male workers. This study showed that almost $4 \%$ of subjects met the criteria for MetS seasonal variation, and MetS diagnosis occurred more often in the winter than in the summer. Current estimates suggest that $\sim 25 \%$ of the world adult population have MetS. Therefore, the choice of the season in which health checkups are conducted may result in overlooking many subjects who should be diagnosed with MetS, which can delay the initiation of therapeutic interventions, such as lifestyle management (for example, weight reduction, smoking cessation, increased physical activity and diet modification). Our data suggest that seasonal variation should be carefully considered in the clinical diagnosis and management of MetS and that MetS screening can be more useful when performed during the winter season. The second important result of the current study is that seasonal variation in MetS prevalence is associated with a mild-to-moderate increase in insulin resistance. This study showed that the prevalence rate of MetS in the third quartile of patients stratified based on HOMA-IR levels was significantly higher in the winter. The frequency of elevated BP in the third quartile was $18.8 \%$ higher in the winter, which correlated with the variation observed in the prevalence data in this study. On the other hand, the decreased variation of the prevalence data in the fourth quartile appeared to be dependent on the severity of the MetS components. These results indicate that increased insulin resistance may be one of the potential mechanisms for seasonal variation of MetS prevalence, and seasonally elevated BP certainly appears to be involved with seasonal variation in MetS. Although the mechanism of seasonal variability in BP is not fully understood, cold temperatures increase the activation of the sympathetic nervous system and the secretion of catecholamines. A large cross-sectional study reported that systolic BP decreases as the air temperature increases with an $8.0 \mathrm{~mm} \mathrm{Hg}$ difference between the lowest and highest air temperature quintiles. ${ }^{29}$ Increased insulin resistance is associated with the activation of not only the sympathetic nervous system but also the renin-angiotensin systems, which could partially contribute to the seasonal variations in BP. Elevated BP has been well characterized as the most common component of MetS and the most attributable risk factor for cardiovascular disease mortality. ${ }^{30-32}$ The INTERSALT study reported that $2.2 \mathrm{~mm} \mathrm{Hg}$ or greater decrease in systolic BP is associated with a $4 \%$ lower risk of coronary death and a $6 \%$ lower risk of stroke-related death in middle-aged adults. ${ }^{33}$ We propose that the strict management of both elevated BP levels and seasonal BP variability has an important role in the prevention of cardiovascular disease. Finally, subjects diagnosed with summer-, winter- and summer-winter-MetS had significantly higher HOMA-IR levels when compared with subjects without MetS. Additionally, HOMAIR levels were lower in subjects with winter-MetS than in subjects with summer- and summer-winter-MetS. The UK Prospective Diabetes Study reported that the incidence of type 2 diabetes was higher during the winter than during other seasons. ${ }^{34}$ These findings indicate that seasonal variation may exist with respect to insulin resistance and that HOMA-IR levels according to MetS prevalence may display more significant seasonal variation in subjects with winter-MetS than in subjects with summer- and summer-winterMetS. Moreover, a recent prospective study showed that even minimal increases in HOMA-IR levels predicted subsequent cardiovascular events in non-diabetic Japanese men. ${ }^{35}$ The results of this study strongly suggest that we should not overlook subjects diagnosed with MetS, regardless of the season.

\section{Study limitations}

First, our study was limited to a single year; therefore, extended observation will be necessary to confirm that the trend remains true. Second, similar studies should be performed using different populations of subjects because the study subjects of the current study may not be representative of all Japanese men. In addition, the results should be confirmed for Japanese female workers because the prevalence rate of MetS in Japanese women is significantly lower when compared with the prevalence rate of MetS in Japanese men. Third, we could not investigate differences in diet, physical activity, climate conditions and mood among the study subjects. To some extent, differences in these factors between the summer and the winter may have influenced the study results. Finally, this study could not demonstrate whether seasonal variation in MetS prevalence actually has a role in type 2 diabetes and cardiovascular disease. We hope that additional studies will be performed in the near future to further our understanding of the clinical role of seasonal variation in MetS prevalence. 


\section{CONCLUSIONS}

We demonstrated that seasonal variation of MetS prevalence was associated with mildly to moderately increased insulin resistance in middle-aged Japanese male workers. This result suggests that increased insulin resistance may be a potential mechanism for the observed seasonal variation in MetS prevalence. Based on the results of this study, seasonal variation in MetS should be taken into account for the clinical diagnosis and management of MetS.

\section{CONFLICT OF INTEREST}

The authors declare no conflict of interest.

\section{ACKNOWLEDGEMENTS}

This study was supported by Grant-in-Aids for Young Scientists (21790755 and 22700706) from the Ministry of Education, Culture, Sports, Science and Technology, Japan. We thank Chizuko Oba for her valuable assistance.

1 Mottillo S, Filion KB, Genest J, Joseph L, Pilote L, Poirier P, Rinfret S, Schiffrin EL, Eisenberg MJ. The metabolic syndrome and cardiovascular risk a systematic review and meta-analysis. J Am Coll Cardiol 2010; 56: 1113-1132.

2 Takeuchi H, Saitoh S, Takagi S, Ohnishi H, Ohhata J, Isobe T, Shimamoto K. Metabolic syndrome and cardiac disease in Japanese men: applicability of the concept of metabolic syndrome defined by the National Cholesterol Education Program-Adult Treatment Panel III to Japanese men-the Tanno and Sobetsu Study. Hypertens Res 2005; 28: 203-208.

3 Grundy SM. Metabolic syndrome pandemic. Arterioscler Thromb Vasc Biol 2008; 28 629-636.

4 Eckel RH, Alberti KG, Grundy SM, Zimmet PZ. The metabolic syndrome. Lancet 2010; 375: $181-183$

5 Alberti KG, Eckel RH, Grundy SM, Zimmet PZ, Cleeman JI, Donato KA, Fruchart JC, James WP, Loria CM, Smith Jr SC. Harmonizing the metabolic syndrome: a joint interim statement of the International Diabetes Federation Task Force on Epidemiology and Prevention; National Heart, Lung, and Blood Institute; American Heart Association; World Heart Federation; International Atherosclerosis Society; and International Association for the Study of Obesity. Circulation 2009; 120: 1640-1645.

6 Yanovski JA, Yanovski SZ, Sovik KN, Nguyen TT, O'Neil PM, Sebring NG. A prospective study of holiday weight gain. N Engl J Med 2000; 342: 861-867.

7 Ockene IS, Chiriboga DE, Stanek 3rd EJ, Harmatz MG, Nicolosi R, Saperia G, Well AD, Freedson P, Merriam PA, Reed G, Ma Y, Matthews CE, Hebert JR. Seasonal variation in serum cholesterol levels: treatment implications and possible mechanisms. Arch Intern Med 2004; 164: 863-870.

8 Kamezaki F, Sonoda S, Tomotsune Y, Yunaka H, Otsuji Y. Seasonal variation in serum lipid levels in Japanese workers. J Atheroscler Thromb 2010; 17: 638-643.

9 Hayashi T, Ohshige K, Sawai A, Yamasue K, Tochikubo O. Seasonal influence on blood pressure in elderly normotensive subjects. Hypertens Res 2008; 31: 569-574.

10 Liang WW. Seasonal changes in preprandial glucose, A1C, and blood pressure in diabetic patients. Diabetes Care 2007; 30: 2501-2502.

11 Carlström M. Seasonal variation may affect clinical diagnosis of metabolic syndrome. Hypertens Res 2010; 33: 531-533.

12 Carlström M. Seasonal variation in metabolic syndrome components: how much do they influence the diagnosis of metabolic syndrome? Curr Cardiovasc Risk Rep 2011 5: 29-37

13 Englund A, Kovanen L, Saarikoski ST, Haukka J, Reunanen A, Aromaa A, Lönnqvist J, T Partonen. NPAS2 and PER2 are linked to risk factors of the metabolic syndrome. J Circadian Rhythms 2009; 7: 5.

14 Grimaldi S, Englund A, Partonen T, Haukka J, Pirkola S, Reunanen A, Aromaa A, Lönnqvist $J$. Experienced poor lighting contributes to the seasonal fluctuations in weight and appetite that relate to the metabolic syndrome. J Environ Public Health 2009; 2009: 165013.
15 Partonen T. Metabolic syndrome follows a seasonal cycle. Hypertens Res 2010; 33: 534.

16 Kobayashi D, Takahashi O, Deshpande GA, Shimbo T, Fukui T. Relation between metabolic syndrome and sleep duration in Japan: a large scale cross-sectional study. Intern Med 2011; 50: 103-107.

17 Rintamäki R, Grimaldi S, Englund A, Haukka J, Partonen T, Reunanen A, Aromaa A, Lönnqvist J. Seasonal changes in mood and behavior are linked to metabolic syndrome. PLOS ONE 2008; 3: e1482.

18 Kamezaki F, Sonoda S, Nakata S, Okazaki M, Tamura M, Abe H, Takeuchi M, Otsuji Y. Elevated depressive symptoms are associated with hypertriglyceridemia in Japanese male workers. Intern Med 2011; 50: 2485-2490.

19 Kamezaki F, Sonoda S, Tomotsune Y, Yunaka H, Otsuji Y. Seasonal variation in metabolic syndrome prevalence. Hypertens Res 2010; 33: 568-572.

20 Kamezaki F, Sonoda S, Nakata S, Kashiyama K, Muraoka Y, Okazaki M, Tamura M, Abe H, Takeuchi M, Otsuji Y. Proposed cutoff level of waist circumference in Japanese men: evaluation by homeostasis model assessment of insulin resistance levels. Intern Med 2012; 51: 2119-2124.

21 Definition and, the diagnostic, standard for, metabolic syndrome. Committee to Evaluate Diagnostic Standards for Metabolic Syndrome. Nihon Naika Gakkai Zassh 2005; 94: 794-809. in Japanese.

22 Matthews DR, Hosker JP, Rudenski AS, Naylor BA, Treacher DF, Turner RC. Homeostasis model assessment: insulin resistance and beta-cell function from fasting plasma glucose and insulin concentrations in man. Diabetologia 1985; 28 412-419.

23 Kang ES, Yun YS, Park SW, Kim HJ, Ahn CW, Song YD, Cha BS, Lim SK, Kim KR, Lee HC. Limitation of the validity of the homeostasis model assessment as an index of insulin resistance in Korea. Metabolism 2005; 54: 206-211.

24 Maury E, Brichard SM. Adipokine dysregulation, adipose tissue inflammation and metabolic syndrome. Mol Cell Endocrinol 2010; 314: 1-16.

25 Kloner RA, Poole WK, Perritt RL. When throughout the year is coronary death most likely to occur? A 12-year population-based analysis of more than 220000 cases. Circulation 1999; 100: 1630-1634.

26 Rumana N, Kita Y, Turin TC, Murakami Y, Sugihara H, Morita Y, Tomioka N, Okayama A, Nakamura $Y$, Ueshima $\mathrm{H}$. Seasonal pattern of incidence and case fatality of acute myocardial infarction in a Japanese population (from the Takashima AMI Registry, 1988 to 2003). Am J Cardiol 2008; 102: 1307-1311.

27 Chen TO. Seasonal variation in serum cholesterol levels may be another explanation for seasonal variation in acute myocardial infarction. Int J Cardiol 2005; 104: 101.

28 Wallace TM, Levy JC, Matthews DR. Use and abuse of HOMA modeling. Diabetes Care 2004; 27: 1487-1495.

29 Alpérovitch A, Lacombe JM, Hanon O, Dartigues JF, Ritchie K, Ducimetière $P_{,}$ Tzourio C. Relationship between blood pressure and outdoor temperature in a large sample of elderly individuals: the Three-City study. Arch Intern Med 2009; 169: 75-80.

30 Conen D, Bamberg F. Noninvasive 24-h ambulatory blood pressure and cardiovascular disease: a systematic review and meta-analysis. J Hypertens 2008; 26: 1290-1299.

31 Shimada K, Fujita T, Ito S, Naritomi H, Ogihara T, Shimamoto K, Tanaka H, Yoshiike N. The importance of home blood pressure measurement for preventing stroke and cardiovascular disease in hypertensive patients: a sub-analysis of the Japan Hypertension Evaluation with Angiotensin II Antagonist Losartan Therapy (J-HEALTH) study, a prospective nationwide observational study. Hypertens Res 2008; 31: 1903-1911.

32 Shimamoto K, Fujita T, Ito S, Naritomi H, Ogihara T, Shimada K, Tanaka H, Yoshiike NJ-HEALTH Study Committees. Impact of blood pressure control on cardiovascular events in 26,512 Japanese hypertensive patients: the Japan Hypertension Evaluation with Angiotensin II Antagonist Losartan Therapy (J-HEALTH) study, a prospective nationwide observational study. Hypertens Res 2008; 31: 469-478.

33 Stamler J, Rose G, Stamler R, Elliott P, Dyer A, Marmot M. INTERSALT study findings. Public health and medical care implications. Hypertension 1989; 14: 570-577.

34 UK Prospective Diabetes Study. V. Characteristics of newly presenting type 2 diabetic patients: estimated insulin sensitivity and islet 4-cell function. Multi-centre study. Diabet Med 1988; 5: 444-448.

35 Nakamura K, Sakurai M, Miura K, Morikawa Y, Ishizaki M, Yoshita K, Kido T, Naruse Y, Nakagawa $\mathrm{H}$. Homeostasis model assessment of insulin resistance and the risk of cardiovascular events in middle-aged non-diabetic Japanese men. Diabetologia 2010 53: $1894-1902$. 